\title{
Bound states in the continuum in symmetric and asymmetric photonic crystal slabs
}

\author{
Anton I. Ovcharenko, ${ }^{1}$ Cédric Blanchard, ${ }^{2}$ Jean-Paul Hugonin, ${ }^{1}$ and Christophe Sauvan ${ }^{1, *}$ \\ ${ }^{1}$ Laboratoire Charles Fabry, Institut d'Optique Graduate School, CNRS, Université Paris-Saclay, 91127 Palaiseau, France \\ ${ }^{2}$ CEMHTI, CNRS UPR 3079, Orléans University, Orléans, France
}

(Received 8 November 2019; accepted 18 March 2020; published 10 April 2020)

\begin{abstract}
We develop a semianalytical model to describe bound states in the continuum (BICs) in photonic crystal slabs. We model leaky modes supported by photonic crystal slabs as a transverse Fabry-Perot resonance composed of a few propagative Bloch waves bouncing back and forth vertically inside the slab. This multimode FabryPerot model accurately predicts the existence of BICs and their positions in the parameter space. We show that, regardless of the slab thickness, BICs cannot exist below a cutoff frequency, which is related to the existence of the second-order Bloch wave in the photonic crystal. Thanks to the semianalyticity of the model, we investigate the dynamics of BICs with the slab thickness in symmetric and asymmetric photonic crystal slabs. We evidence that, as the horizontal mirror symmetry is broken, the symmetry-protected BICs that exist in symmetric structures at the $\Gamma$ point of the dispersion diagram become resonance-trapped BICs, but only for specific values of the slab thickness.
\end{abstract}

DOI: 10.1103/PhysRevB.101.155303

\section{INTRODUCTION}

Photonic crystals $(\mathrm{PhCs})$ consist of a periodic modulation of the refractive index at the wavelength scale [1,2] and $\mathrm{PhC}$ slabs are formed by etching a one- or two-dimensional (1D or $2 \mathrm{D}) \mathrm{PhC}$ in a dielectric layer acting as an optical waveguide. Compared to three-dimensional (3D) $\mathrm{PhCs}$, the simplified architecture of $\mathrm{PhC}$ slabs makes them attractive for on-chip integrated photonics [3,4]. In addition, their peculiar diffraction properties have been used in a wide variety of applications, including filters [5-8], vertical-cavity surfaceemitting lasers (VCSEL) [9-11], photovoltaics [12,13], thermal emission [14], and structural color generation [15].

It was recently pointed out that $\mathrm{PhC}$ slabs can support optical bound states in the continuum (BICs) [16-18]. A BIC (also called embedded eigenvalue $[19,20]$ ) is a bound state that exists at the same energy level as a continuum of radiation modes [21,22]. In PhC slabs, it corresponds to an eigenmode that is truly guided (no radiative leakage) despite the fact that it lies above the light cone in the dispersion diagram $\omega=f(\mathbf{k})$, with $\omega$ the angular frequency and $\mathbf{k}$ the wave vector. The absence of leakage originates from two different physical mechanisms: symmetry incompatibility for so-called symmetry-protected BICs or destructive interference between different leakage channels for so-called resonance-trapped or Friedrich-Wintgen BICs [16,18,22].

From a strictly theoretical point of view, BICs, and especially the ones resulting from an interference mechanism, are definitely counterintuitive and intriguing modes. From a practical point of view, however, BICs do not really exist. Indeed, in a real nonideal structure, they are anyway faintly coupled to the radiation continuum because of technological imperfections, roughness, or finite size of the device. A BIC thereby becomes a leaky mode with extremely low leakage,

*Corresponding author: christophe.sauvan@institutoptique.fr i.e., with a very large quality factor $Q$. Therefore, if a $\mathrm{PhC}$ slab can be fabricated with geometrical parameters close enough to the ideal ones, it exhibits a very sharp resonance with an extremely high-quality factor whose value is only limited by technological constraints. Such high- $Q$ resonances that result from the existence of a BIC nearby in the parameter space have been recently investigated [23-25] and exploited for lasing [26,27] and sensing applications [28-30].

Up to now, the existence of BICs and their location in the parameter space has been calculated either with rigorous numerical methods [16,26,31-35] or with various perturbation approaches based on coupled-wave theory [17,36-39]. Fully numerical approaches are cumbersome even for simple geometries since the whole parameter space has to be explored blindly to find a BIC. Moreover, this exploration has to be performed with a very fine grid since the signature of a BIC in the parameter space is often very narrow. Using a perturbation approach is an interesting alternative. However, if perturbation theories are accurate for $\mathrm{PhC}$ slabs with a low refractive-index contrast, the accuracy drops as the contrast increases. Iterative schemes have been proposed to improve the accuracy of the coupled-wave formalism for high refractive-index contrasts but at the cost of a drastic loss in simplicity [37].

To ease the practical implementation of PhC slabs supporting BICs, in particular with semiconductor materials, one needs approximate models that yield fast yet accurate predictions of the BIC location in the parameter space. Improving the understanding of the physical mechanisms that lead to the BIC formation is also an important issue. We propose a semianalytical model that does not rely on a perturbative approach. The model presents several advantages. First, it explicitly contains the interference mechanism that leads to the formation of a BIC. Second, it yields quantitative predictions of the corresponding optogeometric parameters for any refractive-index contrast.

BICs in PhC slabs are leaky modes whose radiative leakage vanishes for a particular set of parameters. Their modeling 
is intrinsically linked to the phenomenon of guided-mode resonance, which corresponds to the resonant excitation of a leaky mode. Over the years, guided-mode resonance has been described by several theoretical formalisms, such as, for instance, coupled-wave theory [40], perturbation methods [41], or polology framework $[42,43]$. Of particular interest to the objectives of this work is an approach that consists of modeling the reflection and transmission of a PhC slab as a transverse Fabry-Perot resonance composed of a small number of Bloch waves (BWs) bouncing back and forth inside the slab. This approach has been first proposed to interpret qualitatively the high reflectivity of $\mathrm{PhC}$ slabs $[44,45]$. It has been made quantitative in 2006 thanks to the rigorous calculation of the reflectivities of each BW [46]. Further works have then used this multimode Fabry-Perot model to study the optical properties of high-contrast gratings (HCGs) [47-49].

In this paper, we apply the model to the calculation of the dispersion curve and the quality factor of leaky modes supported by $1 \mathrm{D} \mathrm{PhC} \mathrm{slabs.} \mathrm{In} \mathrm{contrast} \mathrm{to} \mathrm{previous} \mathrm{works,} \mathrm{we}$ show that a PhC slab with $N=2$ or 3 propagative BWs can be described by a monomode Fabry-Perot resonator with an effective reflection coefficient that includes the interference effects between the BWs. Introducing an effective reflection allows us to introduce more analyticity in the calculation and to provide closed-form expressions for the dispersion curve and the quality factor of the leaky modes. We show that the semianalytical model is able to quantitatively predict the appearance of BICs and their position in the parameter space. Note that a different two-wave Fabry-Perot model has recently been used to study BICs in partially etched 1D PhC slabs [50]. This model focuses on the plane waves propagating back and forth inside the unetched fraction of the slab whereas the multimode Fabry-Perot model that we use here relies on the BWs that propagate in the $\mathrm{PhC}$.

Let us emphasize that the multimode Fabry-Perot model is particularly well suited for the study of BICs. First, it is derived from a rigorous theory simply by neglecting evanescent waves. Therefore, the BWs used in the model to build the transverse resonance are neither virtual intermediary means for the calculation nor waves propagating in an unperturbed reference structure. They are the waves that propagate in the real structure, even in the case of structures far from the perturbation regime. They exactly correspond to the channels that destructively interfere to form a BIC. Second, the BWs are bouncing back and forth vertically inside the PhC slab. The slab thickness is thus a crucial parameter to understand the formation of BICs by destructive interference. The model predictions are analytical with respect to this geometrical parameter. Third, the BWs possess cutoff frequencies below which they cannot propagate. Since the multimode Fabry-Perot model explicitly contains these cutoff frequencies, the zones in the $(\omega, \mathbf{k})$ space where BICs of different composition, judging by the number of constituent BWs, can exist become apparent with very few calculations. In particular, the model allows us to evidence the existence of a cutoff frequency (related to the secondorder BW) below which no BIC can exist, regardless of the slab thickness.

We apply the multimode Fabry-Perot model to 1D PhC slabs that are either symmetric or asymmetric with respect to (a) air
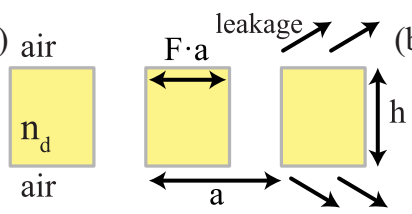

(b) $5 / 6 \mathrm{~F} \cdot \mathrm{a}$

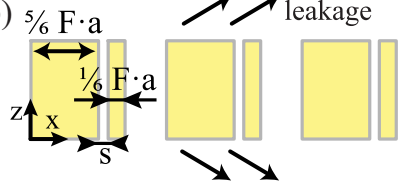

FIG. 1. Schematics of both systems under study: a symmetric (a) and an asymmetric (b) 1D PhC slab. Main parameters are the $\mathrm{PhC}$ period $a$, the filling factor $F$, defined as the fraction of dielectric material inside one period, the refractive index $n_{d}$, and the slab thickness $h$. Throughout the paper, we consider $n_{d}=3.5$, which is close to the refractive index of silicon at $\lambda=1.5 \mu \mathrm{m}$. For the asymmetric PhC slab, a slit of width $s$ is introduced as the asymmetry parameter (no asymmetry for $s=0$ ) with a constant filling factor $F$.

a vertical axis ( $z$ axis in this work) (see Fig. 1). The existence of BICs in asymmetric structures is scarcely documented in the literature [32,51]. Our model by its nature does not discriminate between symmetric or asymmetric slabs; the physical interpretation and the computational cost remain the same as symmetry is broken. Thus, we still benefit from the semianalytical character of the model and we can quickly explore the parameter space. We evidence the existence of BICs in 1D $\mathrm{PhC}$ slabs with a broken horizontal symmetry and we study their evolution as the asymmetry parameter is tuned continuously. We show that, as the horizontal mirror symmetry is broken, the symmetry-protected BICs that exist in symmetric structures at the $\Gamma$ point of the dispersion diagram become resonance-trapped BICs, but only for specific values of the slab thickness.

We first calculate and describe in Sec. II the different types of BICs that can exist in a symmetric 1D PhC slab [see Fig. 1(a)]. These different BICs have already been discussed separately in the literature. Here, we show that all of them can exist together in a given geometry. Moreover, this detailed description allows us to set the foundations for further discussion. In Sec. III, we present the multimode Fabry-Perot model and we show that it can accurately predict the existence of all types of BICs. We also discuss some limitations of the model. We finally apply the model in Secs. IV and V to study the dynamics of BICs with the slab thickness and the existence of BICs in PhC slabs with a broken horizontal symmetry [see Fig. 1(b)]. Section V concludes the work.

\section{SYMMETRIC ONE-DIMENSIONAL PHOTONIC CRYSTAL SLABS}

We consider a symmetric lamellar 1D PhC slab, that is a periodic array of slits in a dielectric membrane with refractive index $n_{d}=3.5$ embedded in air, as shown in Fig. 1(a). The $\mathrm{PhC}$ period, the membrane thickness, and the filling factor in dielectric material are, respectively, denoted with $a, h$, and $F$. We study the leaky modes supported by this structure in transverse electric (TE) polarization, i.e., with an electric field polarized along the slits in the $y$ direction.

The eigenmodes of the PhC slab are characterized by a wave vector $\mathbf{k}=\left(k_{x}, k_{y}\right)$ and an eigenfrequency $\tilde{\omega}=2 \pi c / \tilde{\lambda}$. Because of radiative leakage, for modes with a real wave vector located above the light cone, the eigenfrequencies are complex with a nonzero imaginary part. The latter is related 


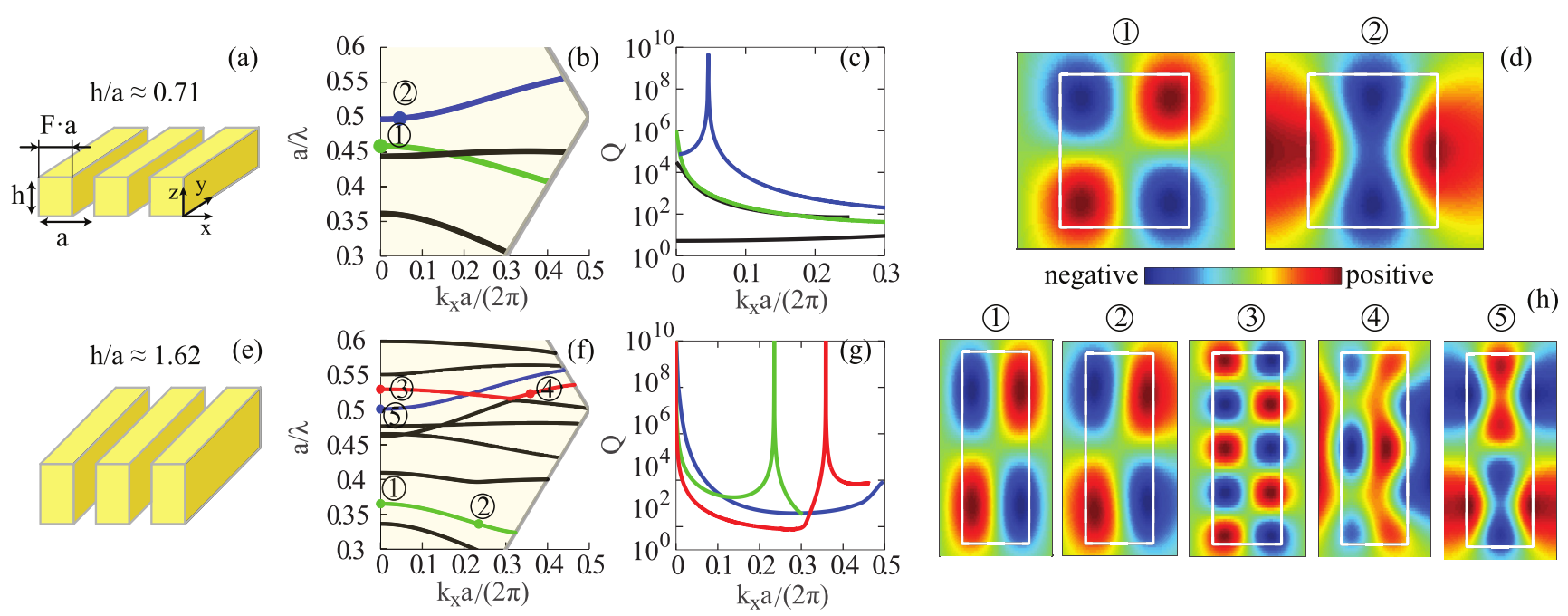

FIG. 2. BICs in symmetric PhC slabs for two different values of the slab thickness, $h=0.71 a$ (a)-(d) and $h=1.62 a$ (e)-(h). (a), (e) Schematics of the structure. The filling factor and the refractive index of the dielectric material are fixed, $F=0.6$ and $n_{d}=3.5$. (b), (f) Dispersion diagrams of the leaky modes above the light cone (gray line). The bands represented with colored curves exhibit BICs for some particular values of the wave vector $k_{x}$ shown by colored dots. (c), (g) Quality factors $Q$ of the leaky modes with the same colors as the dispersion diagram. BICs correspond to $Q$ factors that tend to infinity (numerically larger than $10^{9}$ ). (d), (h) Real part of the electric field $E_{y}(x, z)$ for the BICs shown by colored dots in (b) and (f). A single period is represented; edges of the dielectric ridge are shown with white lines.

to the mode quality factor $Q=\operatorname{Re}(\tilde{\lambda}) /[2 \operatorname{Im}(\tilde{\lambda})]$. Numerical calculations in this work are performed with the rigorous coupled-wave analysis (RCWA) [52]. The leaky modes (or quasinormal modes) of the $\mathrm{PhC}$ slab are calculated by searching for the poles of the scattering matrix in the complex frequency plane $[53,54]$. The number of Fourier harmonics retained in the expansion of the electromagnetic field is $2 M+$ 1 with $M=30$.

Figure 2 shows the different types of BICs that can exist in a symmetric $1 \mathrm{D} \mathrm{PhC} \mathrm{slab.} \mathrm{It} \mathrm{also} \mathrm{evidences} \mathrm{the} \mathrm{crucial} \mathrm{role}$ of the slab thickness in the formation of BICs. Figure 2(b) displays the dispersion curves of the four leaky modes with the lowest frequency for $h=0.71 a$ and $F=0.6$. The normalized frequency $a / \operatorname{Re}(\tilde{\lambda})$ of the modes has been calculated as a function of the normalized $x$ component of the wave vector $k_{x} a /(2 \pi)$, which is varied inside the first Brillouin zone, for a fixed $k_{y}=0$ (nonconical mount). The quality factors of the four modes are shown in Fig. 2(c). Two modes (green and blue curves) exhibit a BIC along their dispersion curve while the other two (black curves) do not. Indeed, the quality factor of the green mode diverges for $k_{x}=0$ and that of the blue mode diverges for $k_{x}=0.046(2 \pi / a)$. The locations of these two BICs in the dispersion diagram are shown with dots labeled (1) and (2) in Fig. 2(b). The corresponding electric fields (real parts) are displayed in Fig. 2(d).

The existence of the BIC labeled (1) at $k_{x}=0$ ( $\Gamma$ point) can be easily understood: radiative leakage is prohibited due to symmetry incompatibility. The field profile of the mode is antisymmetric with respect to $x, E_{y}(-x, z)=-E_{y}(x, z)$, and it cannot couple to the symmetric profile of a plane wave with $k_{x}=0$. These nonleaky modes had been identified in earlier works on PhC slabs without referring to them as BICs. In recent literature they are often defined as symmetry-protected BICs $[16,18]$.
The existence of the BIC labeled (2) is more intriguing. Since $k_{x} \neq 0$, its field does not present any symmetry, even if it looks almost symmetric. It should, in principle, be coupled to the radiation continuum. However, radiative leakage is exactly suppressed at $k_{x}=0.046(2 \pi / a)$. This "accidental" disappearance of leakage results from destructive interference between several leakage channels [16]. In the literature, this type of BIC is referred to as resonance-trapped BIC $[18,26]$ or Friedrich-Wintgen BIC [22].

As the slab thickness increases to $h=1.62 a$ (same filling factor $F=0.6$ ), more modes appear in the spectral range of interest and the number of BICs increases as well [see Figs. 2(e)-2(h)]. Our calculations show five BICs whose locations in the dispersion diagram of Fig. 2(f) are marked with the dots labeled from (1) to (5). The corresponding electric fields are shown in Fig. 2(h). Figure 2(g) displays the quality factors of the three leaky modes that exhibit one or two BICs along their dispersion curve. The green mode has a diverging quality factor at $k_{x}=0$ and another one at $k_{x}=0.235(2 \pi / a)$. The first one is a symmetry-protected BIC with an antisymmetric field profile (1) while the second one is a resonance-trapped BIC with a field profile (2) that is almost antisymmetric but not fully. Similarly, the red mode is a symmetry-protected BIC at $k_{x}=0$ [antisymmetric field profile (3)] and a resonancetrapped $\mathrm{BIC}$ at $k_{x}=0.3587(2 \pi / a)$ [field profile (4)]. Finally, the blue mode exhibits a BIC at $k_{x}=0$. Since its field profile (5) is symmetric, it cannot be a symmetry-protected BIC but rather a resonance-trapped BIC resulting from destructive interference.

So far, we can observe that the seven BICs in Fig. 2 belong to four different families. BICs (1) in Figs. 2(d) and 2(h) and BIC (3) in Fig. 2(h) are symmetry-protected BICs at $k_{x}=0$ with an antisymmetric field profile; BIC (5) in Fig. 2(h) is a resonance-trapped $\mathrm{BIC}$ at $k_{x}=0$ with a symmetric field 
TABLE I. Classification of BICS in symmetric 1D PhC slabs according to the number and symmetry of the Bloch waves that interfere to form the BIC. The table also provides the resulting symmetry properties along the horizontal $x$ axis. The symmetryprotected BICs that result from symmetry mismatch necessarily have an antisymmetric field profile at $k_{x}=0$ and cannot exist at $k_{x} \neq 0$. The resonance-trapped BICs formed by destructive interference can exist equally at $k_{x}=0$ (with a symmetric field profile) or at $k_{x} \neq$ 0 . An antisymmetric (respectively, symmetric) field corresponds to $E_{y}(-x, z)=-E_{y}(x, z)$ [respectively $E_{y}(-x, z)=E_{y}(x, z)$ ]. By quasisymmetric (respectively quasiantisymmetric), we mean that the field profile is almost symmetric (respectively almost antisymmetric), see BICs labeled 2 and 4 in Fig. 2(h) and BIC labeled 2 in Fig. 2(d).

\begin{tabular}{|c|c|c|c|c|}
\hline \multicolumn{2}{|c|}{$\begin{array}{c}\text { Symmetry-protected } \\
\text { BICs }\end{array}$} & \multicolumn{3}{|c|}{$\begin{array}{c}\text { Resonance-trapped } \\
\text { BICs }\end{array}$} \\
\hline$k_{x}=0$ & $k_{x} \neq 0$ & $k_{x}=0$ & & $\neq 0$ \\
\hline Antisym & & Sym & Quasisym & Quasiantisym \\
\hline 2nd BW & & $\begin{array}{c}\text { 3rd BW } \\
+1 \text { st BW } \\
-\end{array}$ & $\begin{array}{c}\text { 3rd BW } \\
+1 \mathrm{st} \mathrm{BW} \\
+2 \mathrm{nd} \mathrm{BW}\end{array}$ & $\begin{array}{c}\text { 2nd BW } \\
+1 \text { st BW } \\
-\end{array}$ \\
\hline
\end{tabular}

profile; BIC (2) in Fig. 2(d) is a resonance-trapped BIC at $k_{x} \neq 0$ with a field profile, which is almost symmetric; BICs (2) and (4) in Fig. 2(h) are resonance-trapped BICs at $k_{x} \neq 0$ with a field profile that is almost antisymmetric. The usual classification with two categories (symmetry-protected and resonance-trapped BICs) is clearly not sufficient to fully characterize BICs in $1 D$ PhC slabs. We summarize in Table I the four different types of BICs that can be inferred from Figs. 2(f)-2(h). We will see in Sec. III that the multimode Fabry-Perot model provides a clear physical understanding of the differences between the four types of BICs by considering the number and the symmetry of the Bloch waves that compose the BIC.

First, as already discussed in the literature, BICs can be separated in two families: symmetry-protected BICs and resonance-trapped BICs. For the first ones, leakage is forbidden because of symmetry incompatibility between the mode of the $\mathrm{PhC}$ slab and the radiative plane wave. These BICs can only exist at $k_{x}=0$; they have necessarily an antisymmetric field profile. Their existence does not depend on the geometrical parameters of the $\mathrm{PhC}$ slab, provided that the horizontal symmetry is conserved. Second, leakage can also be suppressed by destructive interference, resulting in the appearance of so-called resonance-trapped BICs. Figure 2 evidences that this BIC family can be split in three different sub-categories. Resonance-trapped BICs can exist at $k_{x}=0$ with a symmetric field profile or at $k_{x} \neq 0$. In the latter case, the field profile presents no strict symmetry, but it is either quasisymmetric or quasiantisymmetric. By the prefix "quasi," we mean that the symmetry or antisymmetry property is almost fulfilled but not fully [see, for instance, BIC (2) in Fig. 2(h) that is very close to be antisymmetric or BIC (2) in Fig. 2(d) that is very close to be symmetric]. In contrast to the symmetry-protected BICs, the existence of resonance-trapped BICs formed by destructive interference strongly depends on the geometrical parameters. It is thus difficult to predict their precise position along the dispersion curve.

In the following section, we present a multimode FabryPerot model that allows for a simple yet quantitative analysis of the interference mechanism between a small number of BWs composing a leaky mode. Thanks to its semianalytical character, the model allows for easy calculations of the BIC positions in the dispersion diagram and their variation as a function of the slab thickness.

\section{MULTIMODE FABRY-PEROT MODEL}

We derive a semianalytical model that predicts the dispersion curve and the quality factor of leaky modes supported by a PhC slab. We extend an approach proposed in Ref. [46] for the calculation of the reflection and transmission of a $\mathrm{PhC}$ slab. A leaky mode is nothing but a transverse Fabry-Perot resonance composed of several Bloch waves (BWs) bouncing back and forth vertically inside the slab. This description is perfectly rigorous as long as a sufficiently large number $M$ of waves are taken into account. This is the mathematical ground of RCWA, also known as the Fourier modal method [52]. In the case of subwavelength periodic structures, only a small number $N$ of BWs are propagative, the other ones being evanescent [46,55]. Neglecting the impact of the evanescent waves provides approximate closed-form expressions that can be very accurate, provided that the slab thickness is large enough, typically larger than the decay length of the least attenuated evanescent wave [46].

In the following, we first introduce some notations and write the general equations that lead to an exact calculation of the leaky modes dispersion. We then neglect the evanescent BWs and derive closed-form expressions of the dispersion curve and the quality factor for $N=1,2$, and 3 propagative BWs. We evidence that BICs can only exist when at least two BWs are propagative. It sets a cutoff frequency below which no BIC can exist whatever the value of the slab thickness. We finally validate the model by comparing its semianalytical predictions to exact calculations.

\section{A. Notations and general equations}

Before building a multimode Fabry-Perot resonance in a $\mathrm{PhC}$ slab of thickness $h$, we need to solve the problem of a single interface between a semi-infinite $\mathrm{PhC}$ and a semiinfinite homogeneous medium [see Fig. 3(a)]. We denote by $\beta_{i}$ the propagation constant of the $i$ th $\mathrm{BW}$ along the vertical $z$ direction. In a nonabsorbing $\mathrm{PhC}, \beta_{i}$ is either purely real (propagative wave) or purely imaginary (evanescent wave). The number of propagative BWs depends on the geometry. For example, for $k_{x}=0, F=0.6$, and $n_{d}=3.5$, only up to three BWs are propagative in the band $a / \lambda<0.6$. Their propagation constants are shown in Fig. 3(b), where we can observe the second and third BW cutoffs at $a / \lambda=0.327$ and 0.45 , respectively. Note that the fundamental BW (largest propagation constant, blue curve) has no cutoff and is propagative regardless of the $a / \lambda$ value. The corresponding field profiles $E_{y}(x)$ are shown in Fig. 3(c). The fundamental BW is symmetric and the higher-order BWs have alternately an antisymmetric or a symmetric field profile. For $k_{x} \neq 0$, the 
(a)

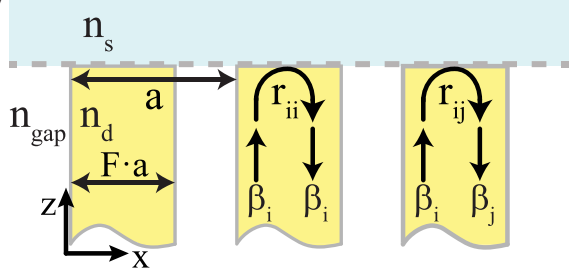

(d)

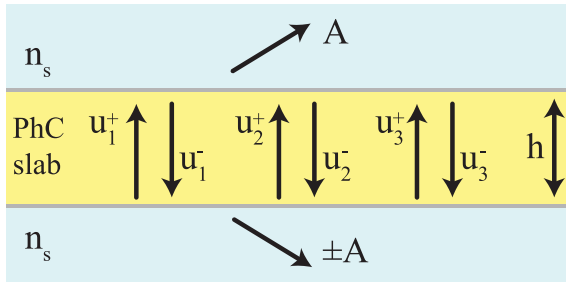

(b)

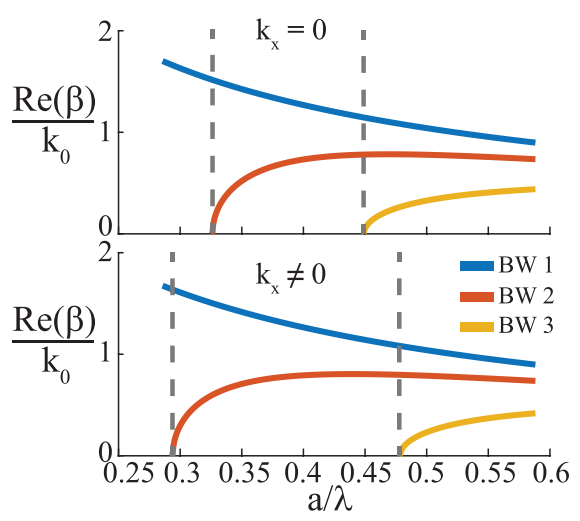

(c)

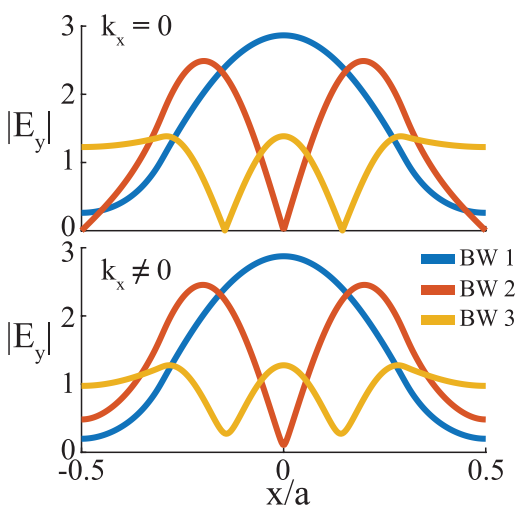

FIG. 3. Multimode Fabry-Perot model. (a) Interface between a semi-infinite PhC and a homogeneous medium. The refractive index of the homogeneous medium $n_{s}$ can be different from the index $n_{\text {gap }}$ inside the slits of the PhC. At the interface, the Bloch waves (characterized by their propagation constants $\beta_{i}$ ) propagating in the periodic medium are reflected with a reflection coefficient $r_{i i}$, cross reflected with a reflection coefficient $r_{i j}$, or transmitted with a transmission coefficient $t_{i}$. (b) Normalized propagation constants of the BWs for $F=0.6$ as a function of the frequency at the $\Gamma$ point $\left(k_{x}=0\right)$ and at $k_{x}=0.2 a /(2 \pi)$. The cutoff frequencies of the second and third BWs are shown by vertical dashed lines. (c) Electric-field profile $\left|E_{y}(x)\right|$ of the three propagative BWs for $a / \lambda=0.526$. (d) Principle of the multimode Fabry-Perot model. In the spectral range of interest, up to three BWs can propagate back and forth inside the PhC slab, all other BWs being evanescent. Each BW is transmitted in the surrounding medium with its own phase. The amplitude $A$ of the plane wave propagating away (radiative leakage) results from the interference between these three contributions.

cutoff frequencies vary with $k_{x}$ and the BWs are no longer strictly symmetric nor antisymmetric.

As the $i$ th BW is incident on an interface with a homogeneous medium, it is reflected with a reflection coefficient $r_{i i}$. In addition, it is reflected into a different $\mathrm{BW}$ with a cross-reflection coefficient $r_{i j}$, and transmitted as a propagative plane wave with a transmission coefficient $t_{i}$. The coefficients $r_{i i}, r_{i j}$, and $t_{i}$ are the generalized Fresnel coefficients for an interface between a homogeneous and a periodic media. Note that we limit ourselves to the case where a single plane wave is propagative in the homogeneous medium, the zeroth diffraction order of the PhC slab. The energy contained in this plane wave corresponds to the radiative leakage.

In a PhC slab of thickness $h$, the BWs are reflected at the top and bottom interfaces. Thus, they propagate back and forth inside the slab as illustrated in Fig. 3(d). We denote by $u_{i}^{+}$and $u_{i}^{-}$the amplitudes of the up- and down-propagating $i$ th wave, respectively. The phase origin for the amplitude $u_{i}^{+}$ (respectively $u_{i}^{-}$) is taken at the bottom interface (respectively the top interface).

Leaky modes of a PhC slab are solutions of Maxwell's equations in the absence of an incident wave. If one considers a finite number $M$ of BWs (propagative and evanescent BWs), the amplitudes $u_{i}^{+}$and $u_{i}^{-}$are related by

$$
\begin{aligned}
& u_{i}^{+}=\sum_{j=1}^{M} r_{j i} u_{j}^{-} \exp \left(i \beta_{j} h\right), \\
& u_{i}^{-}=\sum_{j=1}^{M} r_{j i} u_{j}^{+} \exp \left(i \beta_{j} h\right) .
\end{aligned}
$$

The number $M$ of BWs is equal to the truncation rank of the Fourier series in RCWA [52], $M=30$ for the calculations shown in Fig. 2. For the sake of simplicity, we consider a PhC slab surrounded by the same homogeneous medium above and below. The equations can be straightforwardly generalized to the case of two different media (e.g., for a PhC slab lying over a substrate [56]); two different families of reflection coefficients $r_{j i}^{T}$ and $r_{j i}^{B}$ have to be considered [57].

Equations (1) can be rewritten in a matrix form

$$
\mathbf{R}\left(k_{x}, \lambda\right) \mathbf{U}=0
$$

where the vector $\mathbf{U}$ is built with the amplitudes $u_{i}^{+}$and $u_{i}^{-}$, $\mathbf{U}=\left[u_{1}^{+}, u_{1}^{-}, \ldots, u_{M}^{+}, u_{M}^{-}\right]^{t}$, and the matrix $\mathbf{R}\left(k_{x}, \lambda\right)$ contains all reflections and cross-reflection coefficients. A leaky mode is a nontrivial solution of this linear system of equations; it corresponds to a pair $\left(k_{x}, \tilde{\lambda}\right)$ (with $k_{x}$ a real number and $\tilde{\lambda}$ a complex number) that satisfies [33]

$$
\operatorname{det}\left[\mathbf{R}\left(k_{x}, \tilde{\lambda}\right)\right]=0,
$$

with det being the determinant of a matrix. We calculate rigorously with RCWA the parameters of a single interface $\left(\beta_{i}, r_{i i}, r_{i j}, t_{i}\right)$, and thus the matrix $\mathbf{R}\left(k_{x}, \lambda\right)$, as a function of the wavelength for a fixed value of the wave vector $k_{x}$. Then, Eq. (3) can be solved, typically with an iterative procedure such as the Newton algorithm or a different method using a Padé approximation [53], to find the complex wavelength $\tilde{\lambda}$ of the leaky mode. The dispersion curve and the quality factor are then given, respectively, by $a / \operatorname{Re}(\tilde{\lambda})=f\left(k_{x}\right)$ and $Q=\operatorname{Re}(\tilde{\lambda}) /[2 \operatorname{Im}(\tilde{\lambda})]$. 
Regarding the radiative leakage, the amplitude of the outgoing propagative plane wave is given by

$$
A=\sum_{j=1}^{M} t_{j} u_{j}^{+} \exp \left(i \beta_{j} h\right) .
$$

The radiative leakage results from the interference of the BWs amplitudes being transmitted by the interface. Therefore, the leaky mode of the PhC slab is a BIC if, and only if, the interference is perfectly destructive. One readily realizes the crucial role of the slab thickness $h$ in this mechanism since it drives the value of the phase difference between the different BWs.

Solving Eq. (3) for a large number $M$ of BWs, hence containing a bunch of evanescent waves, yields a rigorous and exact result for the dispersion curve and quality factor. On the other hand, since the period of the $\mathrm{PhC}$ slab is subwavelength, neglecting all the evanescent BWs to keep only a small number $N<M$ of propagative BWs drastically reduces the size of the linear system in Eq. (1). Within this approximation, it is possible to derive closed-form expressions for the dispersion curve, the quality factor $Q$, and the radiative leakage $A$, as shown hereafter. In particular, these expressions provide analytical results with respect to the thickness $h$. Note that each of the $N$ propagative BWs is calculated rigorously with $2 M+1$ Fourier terms.

\section{B. Transverse resonance for $N=1$ wave}

Let us start with the simplest case $N=1$ when a single $\mathrm{BW}$ is propagative inside the $\mathrm{PhC}$ slab, all the other waves being evanescent. Although self-evident, this case allows us to introduce the main equations of the model. The single-mode regime occurs when the period-to-wavelength ratio $a / \lambda$ is small, typically between the limit $a / \lambda \rightarrow 0$ (quasistatic limit) and the cutoff of the second BW. For the example in Fig. 3(b), it corresponds to $a / \lambda<0.272$ for all $k_{x}$ values from zero to the light line.

For a single propagative BW, Eq. (3) simply reduces to the usual resonance condition of a Fabry-Perot resonator

$$
1-r_{11}^{2} \exp \left(2 i \beta_{1} h\right)=0 .
$$

With no further assumption, the complex wavelengths that satisfy Eq. (5) have to be found numerically, typically with an iterative algorithm. In order to derive closed-form expressions of the dispersion curve and most of all of the quality factor, we make two additional assumptions. We assume that (i) the quality factor of the resonance is large $Q \gg 1$, and (ii) the modulus of $r_{11}$ varies slowly with the wavelength over the resonance bandwidth $\partial\left|r_{11}\right| / \partial \lambda \approx 0$. The validity of these assumptions will be discussed in Sec. III E. They are important to really obtain closed-form expressions for the dispersion curve and the quality factor. Without these assumptions, one has to solve Eq. (5) iteratively for each value of the slab thickness and the model is not analytic with respect to $h$.

With these two assumptions, an eigenmode of the $\mathrm{PhC}$ slab corresponds to a $\mathrm{BW}$ that returns in phase after half a round trip $[58,59]$,

$$
\Phi_{T}\left(\lambda_{0}, k_{x}\right)=\beta_{1} h+\arg \left(r_{11}\right)=p \pi,
$$

where $\lambda_{0}=\operatorname{Re}(\tilde{\lambda})$ and $p$ is an integer. The phase $\Phi_{T}$ is the total phase accumulated by the BW after half a round trip inside the slab. This phase-matching condition gives an implicit definition of the dispersion curve. The quality factor $Q$ is given by $[59,60]$

$$
Q=-\frac{\lambda_{0}}{1-\left|r_{11}\right|^{2}} \frac{\partial \Phi_{T}}{\partial \lambda},
$$

where the derivative and the reflection $r_{11}$ are taken at $\lambda=\lambda_{0}$.

Within the Fabry-Perot model with $N=1$ propagative BW, the amplitude $A$ of the radiated plane wave is simply proportional to the BW amplitude inside the slab:

$$
A=t_{1} u_{1}^{+} \exp \left(i \beta_{1} h\right) \text {. }
$$

In this case, the leakage does not result from the interference between several channels. It vanishes if, and only if, the transmission $t_{1}$ is strictly equal to zero. This, however, never happens for symmetry reasons. ${ }^{1}$

The Fabry-Perot model allows us to draw an important conclusion: no BIC can exist at a frequency where a single $B W$ is propagative. This result sets a spectral cutoff to this existence of BICs [see Fig. 3(b)]. We emphasize that this cutoff is independent of the slab thickness.

\section{Transverse resonance for $N=2$ waves}

For larger period-to-wavelength ratios, the second BW, which has an antisymmetric field profile, becomes propagative [see Fig. 3(b)]. Let us start with the situation $k_{x}=0$. Since the fundamental BW is symmetric whereas the second $\mathrm{BW}$ is antisymmetric [see Fig. 3(c)], the cross reflections $r_{12}$ and $r_{21}$ are equal to zero and Eqs. (1) reduce to two uncoupled sets of two equations each. As a consequence, leaky modes result from a transverse resonance built either with the fundamental $\mathrm{BW}$ alone or with the second BW alone. The dispersion curve and the quality factor are given by Eqs. (6) and (7) with either $\left(\beta_{1}, r_{11}\right)$ or $\left(\beta_{2}, r_{22}\right)$.

Because of the symmetry mismatch between the BW and the propagative plane wave, $\left|r_{22}\right|=1$ and $t_{2}=0$. Therefore, the mode of the $\mathrm{PhC}$ slab that corresponds to a transverse resonance built with the second $\mathrm{BW}$ alone is necessarily a BIC whatever the geometrical parameters. In particular, varying the slab thickness $h$ shifts the dispersion curve according to the phase-matching condition but the $Q$ factor remains infinite (since $\left|r_{22}\right|=1$ ) and this mode at $k_{x}=0$ is truly guided with no radiative leakage. It is the aforementioned symmetryprotected BIC, which results from symmetry incompatibility (see Table I).

As we depart from the $\Gamma$ point, the BWs are coupled since $r_{12} \neq 0$ and $r_{21} \neq 0$. As a consequence, Eqs. (1) become a set of four coupled equations and any transverse resonance results from the interplay between both BWs. It is possible to replace such two-wave resonator with the usual single-wave

\footnotetext{
${ }^{1}$ For Bloch-wave-to-plane-wave transmission $t_{i}$ to disappear due to symmetry incompatibility, the Bloch wave must have an antisymmetric field profile at $k_{x}=0$, in contrast to the symmetric profile of the plane wave. Since the fundamental Bloch wave $i=1$ has a symmetric field profile at $k_{x}=0, t_{1}$ has always a nonzero value.
} 
Fabry-Perot by introducing an effective reflection coefficient $r_{\text {eff }}$ [57]. The effective reflection fully includes the impact of the second wave. For $k_{x}=0$, a leaky mode is a purely singlewave transverse resonance built either with the fundamental $\mathrm{BW}$ or with the second $\mathrm{BW}$. When $k_{x}$ becomes nonzero, both BWs are mixed but one keeps a larger contribution than the other. For instance, for a band with a symmetry-protected BIC at $k_{x}=0$ [green curves in Figs. 2(b) and 2(f)], $\left|r_{11}\right|<\left|r_{22}\right|$ and $\left|r_{12}\right|<\left|r_{22}\right|$. In that case, the second BW is dominant and we incorporate the effect of the first BW in the effective reflection coefficient. The resonance condition given by Eq. (3) becomes

$$
1-\left(r_{\text {eff }}^{(12)}\right)^{2} \exp \left(2 i \beta_{2} h\right)=0,
$$

where the effective reflection $r_{\mathrm{eff}}^{(12)}$ is given by

$$
r_{\mathrm{eff}}^{(12)}=\frac{r_{22}+\alpha r_{11} r_{21} r_{12} \exp \left(2 i \beta_{1} h\right)}{1-\alpha r_{21} r_{12} \exp \left[i\left(\beta_{1}+\beta_{2}\right) h\right]},
$$

with $\alpha=\left[1-r_{11}^{2} \exp \left(2 i \beta_{1} h\right)\right]^{-1}$. The superscript (12) stands for the fact that $r_{\text {eff }}^{(12)}$ includes the multiple cross reflections between BWs 1 and 2. Note that for $k_{x}=0$, since $r_{12}=r_{21}=$ 0 , we recover $r_{\text {eff }}^{(12)}=r_{22}$. Details on the derivation of Eq. (9) can be found in the Supplemental Material [56].

We can thus apply the usual equations of a Fabry-Perot resonator provided that $\left|r_{\text {eff }}^{(12)}\right|$ varies smoothly with the wavelength. The dispersion curve and the quality factor of a leaky mode composed of two BWs are given by Eqs. (6) and (7) by replacing $\beta_{1}$ and $r_{11}$ by $\beta_{2}$ and $r_{\text {eff }}^{(12)}$. The amplitude of the radiated plane wave is now given by the superposition of both BWs, $A=t_{1} u_{1}^{+} \exp \left(i \beta_{1} h\right)+t_{2} u_{2}^{+} \exp \left(i \beta_{2} h\right)$. Similarly to the resonance condition, an effective transmission coefficient can be introduced,

$$
A=t_{\mathrm{eff}}^{(12)} u_{2}^{+} \exp \left(i \beta_{2} h\right)
$$

with

$$
t_{\mathrm{eff}}^{(12)}=t_{2}+t_{1} \alpha r_{21} e^{i \beta_{1} h}\left[r_{\mathrm{eff}}^{(12)} e^{i \beta_{2} h}+r_{11} e^{i \beta_{1} h}\right] .
$$

Again, for $k_{x}=0, t_{\mathrm{eff}}^{(12)}=t_{2}$ since $r_{21}=0$. One readily realizes that the effective transmission can be canceled if the second term in Eq. (12) is equal to $-t_{2}$. In that case, both BWs interfere destructively to cancel the overall leakage, leading to the formation of a BIC. Figure 4 illustrates the interference mechanism as a function of $k_{x}$ for different values of the slab thickness. For $k_{x}=0, t_{\mathrm{eff}}^{(12)}=t_{2}=0$ for symmetry reasons. For $k_{x} \neq 0, t_{\text {eff }}^{(12)}$ is largely different from $t_{2}$ due to the impact of the first BW. The second cancellation of $t_{\mathrm{eff}}^{(12)}$ is due to destructive interferences between both BWs. The slab thickness drives the phase difference between both BWs and the wave vector that corresponds to destructive interference increases with $h$.

The multimode Fabry-Perot model allows us to understand that the resonance-trapped BIC labeled (2) in Figs. 2(f)-2(h) is formed by the interference between the first and the second $\mathrm{BW}$, with a dominant contribution from the second BW. It is because the latter has an antisymmetric field profile for $k_{x}=0$ and an almost antisymmetric profile for $k_{x} \neq 0$ that the BIC labeled (2) in Figs. 2(f)-2(h) has a quasiantisymmetric field profile [see Fig. 2(h) and Table I].

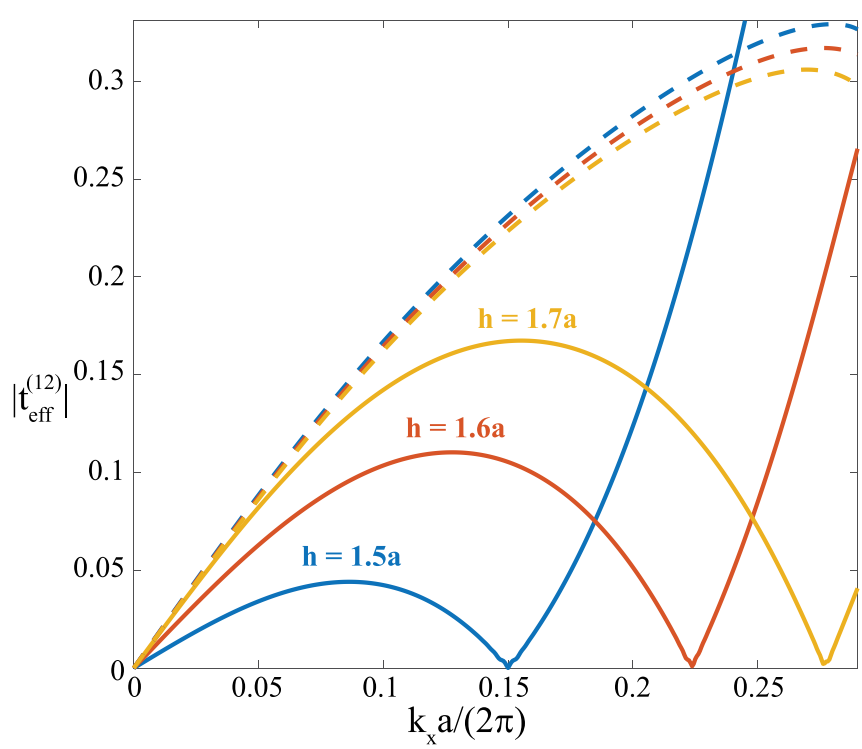

FIG. 4. Effective transmission $t_{\mathrm{eff}}^{(12)}$ (solid lines) for $N=2$ propagative $\mathrm{BWs}$ in a $1 \mathrm{D}$ symmetric $\mathrm{PhC}$ slab with $F=0.6$. The first cancellation of $t_{\text {eff }}$ for $k_{x}=0$ results from symmetry arguments. The second cancellation for $k_{x} \neq 0$ results from destructive interferences between both BWs and varies with the slab thickness. The frequency follows the dispersion curve of the leaky mode shown by the green curve in Fig. 2(f). Dashed lines show the transmission coefficent $t_{2}$ alone and evidence the impact of the first $\mathrm{BW}$ on the radiative leakage.

Note that Eqs (9)-(12) have been written in the case where the second BW is dominant over the first one. In the case where the first $\mathrm{BW}$ is dominant over the second one, we have $1-r_{11}^{2} \exp \left(2 i \beta_{1} h\right) \approx 0$ and the coefficient $\alpha$ becomes extremely large. Calculations are thus more stable if we keep the first BW and incorporate the second BW into the effective reflection. In that case, the subscripts 1 and 2 have simply to be inverted in Eqs. (9) and (10).

\section{Transverse resonance for $N=3$ waves}

As the period-to-wavelength ratio is further increased, the third BW becomes propagative [see Fig. 3(b)]. For $k_{x}=0$ and $F=0.6$, this corresponds to $a / \lambda>0.45$. In that case, Eqs. (1) become a $6 \times 6$ system. Although more tedious, it is still possible to replace the complex interplay between the three BWs by effective reflection and transmission coefficients.

As for $N=2$, let us start the discussion with the case $k_{x}=$ 0 . At the $\Gamma$ point, the first and third BWs have a symmetric field profile while the second BW is antisymmetric. The latter is thus decoupled from BWs 1 and 3. Even if three BWs are propagative, the leaky modes of the $\mathrm{PhC}$ slab are either formed by the second BW alone (symmetry-protected BIC) or by the interplay between first and third BWs. In that case, we can apply the results from previous section by introducing an effective reflection coefficient $r_{\text {eff }}^{(13)}$ instead of $r_{\text {eff }}^{(12)}$. Such a leaky mode formed by BWs 1 and 3 is a BIC if the interference leads to $\left|r_{\text {eff }}^{(13)}\right|=1$ and $t_{\text {eff }}^{(13)}=0$. This is the case of the blue mode labeled (5) in Figs. 2(f)-2(h).

For $k_{x} \neq 0$, the three BWs are coupled and we introduce an effective reflection coefficient $r_{\text {eff }}^{(123)}$, whose closed-form 
expression can be found in the Appendix. Similarly to the $N=2$ case, we keep the BW that has the most important contribution and incorporate the impact of the two other BWs inside the effective reflection. For a band with a symmetryprotected $\mathrm{BIC}$ at $k_{x}=0$, the dominant $\mathrm{BW}$ is the second one; for a band without symmetry-protected BIC at $k_{x}=0$, the dominant BW is usually the third one. In the latter case, the resonance condition given by Eq. (3) simply becomes

$$
1-\left(r_{\mathrm{eff}}^{(123)}\right)^{2} \exp \left(2 i \beta_{3} h\right)=0
$$

where $r_{\text {eff }}^{(123)}$ is an effective reflection coefficient whose expression is given in the Appendix. Details on the derivation of Eq. (13) can be found in the Supplemental Material [56]. We can thus apply the usual equations of a Fabry-Perot resonator, provided that $\left|r_{\text {eff }}^{(123)}\right|$ varies smoothly with the wavelength. The dispersion curve and quality factor of a leaky mode composed of three BWs are given by Eqs. (6) and (7) by replacing $\beta_{1}$ and $r_{11}$ by $\beta_{3}$ and $r_{\text {eff }}^{(123)}$.

The multimode Fabry-Perot model with $N=3$ propagative BWs allows for the understanding of the formation of the symmetric resonance-trapped BICs at $k_{x}=0$ and of the quasisymmetric resonance-trapped BICs at $k_{x} \neq 0$ identified in Table I and Figs. 2. The first ones arise from the interference between two symmetric BWs, the first and third ones, which are not coupled to antisymmetric BWs at $k_{x}=0$. The second ones are formed by the interference between three BWs (first, second, and third) with a dominant contribution from the third $\mathrm{BW}$, whose field profile is almost symmetric for $k_{x} \neq 0$.

Finally, the multimode Fabry-Perot model used either with $N=2$ or 3 propagative BWs allows for the understanding of all four types of BICs observed in Fig. 2 and summarized in Table I.

\section{E. Model validation and discussion}

To validate the multimode Fabry-Perot model, we consider the symmetric 1D PhC slab of Fig. 2(e). We apply the model, either with $N=2$ or $3 \mathrm{BWs}$, and calculate the dispersion curves and quality factors of the different leaky modes supported by the $\mathrm{PhC}$ slab. The model prediction for the dispersion curves (not shown here) and for the $Q$ factors (see Fig. 5) are in quantitative agreement with the rigorous RCWA calculations, which takes into account a large number of evanescent BWs $(M=30)$. It is noteworthy that the semianalytical model accurately predicts all four types of BICs supported by a $1 \mathrm{D}$ symmetric $\mathrm{PhC}$ slab and summarized in Table I.

From a numerical point of view, the analyticity of the multimode Fabry-Perot model relies on Eqs. (6) and (7). In practice, one simply needs to calculate numerically with the RCWA the single-interface quantities defined in Fig. $3\left(\beta_{i}\right.$, $r_{i i}$, and $r_{i j}$ ) over the spectral range of interest. Then, one can apply Eq. (6) for any value of the slab thickness $h$ to find analytically the eigenfrequencies $\lambda_{0}$ for a given value of the integer $p$. Finally, Eq. (7) gives the corresponding quality factor with no further calculations. The model is therefore extremely efficient to investigate the dynamics of BICs with the slab thickness.

The model also provides some physical insight into the nature of the BICs, which before we could only qualitatively

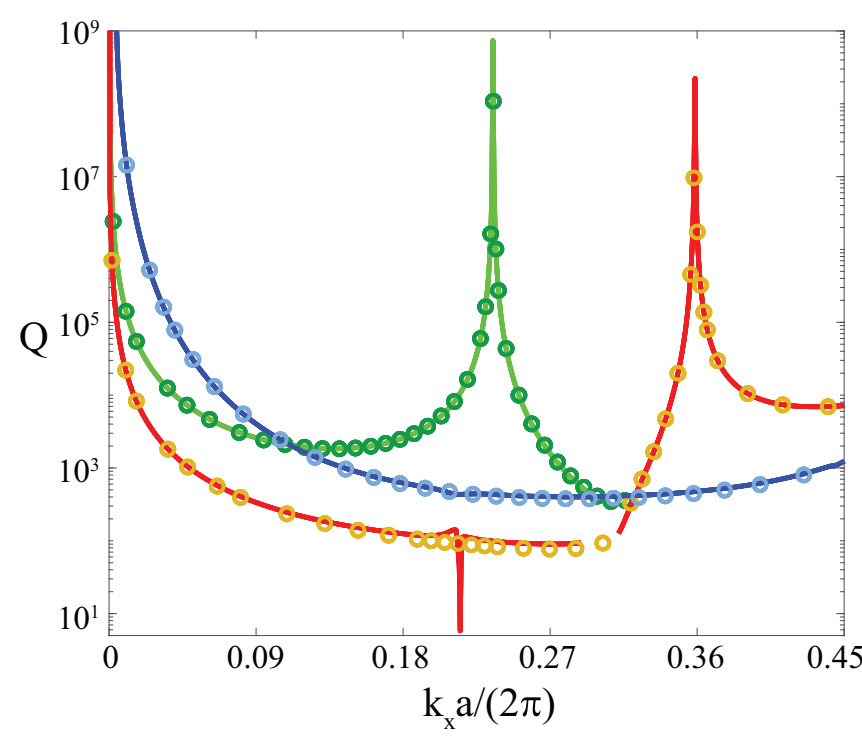

FIG. 5. Quality factors of three leaky modes supported by a 1D symmetric $\mathrm{PhC}$ slab with $n_{d}=3.5, F=0.6$, and $h=1.62 a$ [see Fig. 2(e)]. The predictions of the multimode Fabry-Perot model (solid lines) are in excellent agreement with the exact calculations (markers). The model accurately reproduces the existence and positions of all four types of BICs. The discrepancies between the red curve and the yellow circles for $k_{x} \approx 0.215(2 \pi) / a$ and $k_{x} \approx$ $0.3(2 \pi) / a$ are explained in the text.

infer from the field profiles. The green mode in Fig. 2(f) is mostly given by the second antisymmetric $\mathrm{BW}$, with a small contribution of the fundamental BW for $k_{x} \neq 0$. To calculate it we used the phase-matching condition (9) with $r_{\text {eff }}^{(12)}$ from Eq. (10). The blue mode in Fig. 2(f) is dominated by the third BW with a small impact of the first and second BWs. For this mode, $r_{\text {eff }}^{(123)}$ with the phase factor $\exp \left(i \beta_{3} h\right)$ was used [see Eq. (13)].

We can also assert that the BIC labeled (5) in Fig. 2(f) is fundamentally different from the ones labeled (1) and (3), which are also lying at the $\Gamma$ point. Indeed, it is a resonancetrapped BIC resulting from the destructive interference between the first and third BWs whereas BICs (1) and (3) are symmetry-protected BICs. Similarly, the red leaky mode is also formed by three BWs. However, it couples with a different leaky mode around $k_{x} \approx 0.3(2 \pi) / a$ [see the anticrossing between the red and black dispersion curves in Fig. 2(f)]. Because of this coupling, the red leaky mode changes its symmetry from the quasiantisymmetric one [for $k_{x}<0.3(2 \pi) / a$ ] to the quasisymmetric one [for $k_{x}>0.3(2 \pi) / a$ ], which is reflected in the BIC field profiles labeled (3) and (4) in Fig. 2(h). In this case, different phase-matching equations have to be used for the left and right parts of the dispersion curve. Basically, indices 2 and 3 have to be interchanged in the expression of $r_{\text {eff }}^{(123)}$ and in the phase-matching condition to reflect the fact that on one side of the coupling region the second BW is dominant while on the other side the third $\mathrm{BW}$ is dominant. Then, $r_{\mathrm{eff}}^{(123)}$ becomes equal to $r_{22}$ at $k_{x}=0$ and gives rise to the symmetry-protected BIC labeled (3). In contrast, the other BIC at $k_{x} \approx 0.36(2 \pi) / a$ stems from the 
interference of three BWs, the third (symmetric) being the dominant one.

This aspect of the model can be a source of erroneous results. The algebraic manipulations that lead to the definition of an effective reflection assume that some quantities [such as $\alpha^{-1}$ in Eq. (10)] are different from zero. As a consequence, some roots of the two- or three-wave resonance condition are not contained in the single-wave resonance condition given by Eqs. (9) and (13). The phase-matching condition gives thus a crossing of the dispersion curves instead of an anticrossing. This is the reason why the model fails in Fig. 5 for the red curve around $k_{x} \approx 0.3(2 \pi) / a$. This point is further illustrated in the Supplemental Material [56].

Finally, we would like to clarify the obviously erroneous feature of the red curve around $k_{x} \approx 0.215(2 \pi) / a$. As mentioned in previous sections, the resonance condition and quality factor can be written in the form of Eqs. (6) and (7) only under the assumption that the modulus of the reflection coefficient varies smoothly with the wavelength. However, this assumption is not always valid when we deal with effective reflection coefficients. The appearance of an erroneous resonance in the dispersion curve predicted with the FabryPerot model (see the red solid curve in Fig. 5) is due to this situation: $\left|r_{\text {eff }}^{(123)}\right|$ experiences a sudden resonancelike variation as a function of the wavelength [56] and Eqs. (6) and (7) lose their accuracy.

\section{DYNAMICS OF BICS WITH THE SLAB THICKNESS}

Thanks to the analyticity of the model with respect to the slab thickness, we can apply it for a large number of $h$ values to observe the dynamics of BICs with no additional RCWA calculations. Figure 6 shows the variation of the quality factor of a leaky mode of the $\mathrm{PhC}$ slab, in the spectral range between the second and third BW cutoffs, as a function of the thickness $h / a$ and the wave vector $k_{x} a /(2 \pi)$. We have chosen to follow the dynamics of the mode represented with the green curve in Figs. 2(f) and 2(g).

We can see three branches (shown by white arrows) where $r_{\text {eff }}^{(12)}$ equals exactly 1 , leading to an infinite- $Q$ factor. The first branch is vertical at $k_{x}=0$ and corresponds to the symmetryprotected BIC, which exists regardless of the value of $h$. The two other branches show the $\left(h, k_{x}\right)$ values for which the interference between the first and second BWs is perfectly destructive and results in the formation of a resonancetrapped BIC. The dashed lines show the positions of similar resonance-trapped BICs for $p=0$ in Eq. (6). They belong to the dispersion curve of a different leaky mode. For similar results in the spectral band where three BWs are propagative, we refer the reader to the Supplemental Material [56].

\section{ASYMMETRIC ONE-DIMENSIONAL PHOTONIC CRYSTAL SLABS}

We finally use the multimode Fabry-Perot model to study the behavior of the BICs under broken mirror symmetry. We consider a $1 \mathrm{D} \mathrm{PhC}$ slab with a vertical mirror symmetry but no horizontal mirror symmetry as depicted in Fig. 1(b). The asymmetry parameter $s$ is the size of the air gap that divides the dielectric ridge into a bigger and a smaller parts of sizes

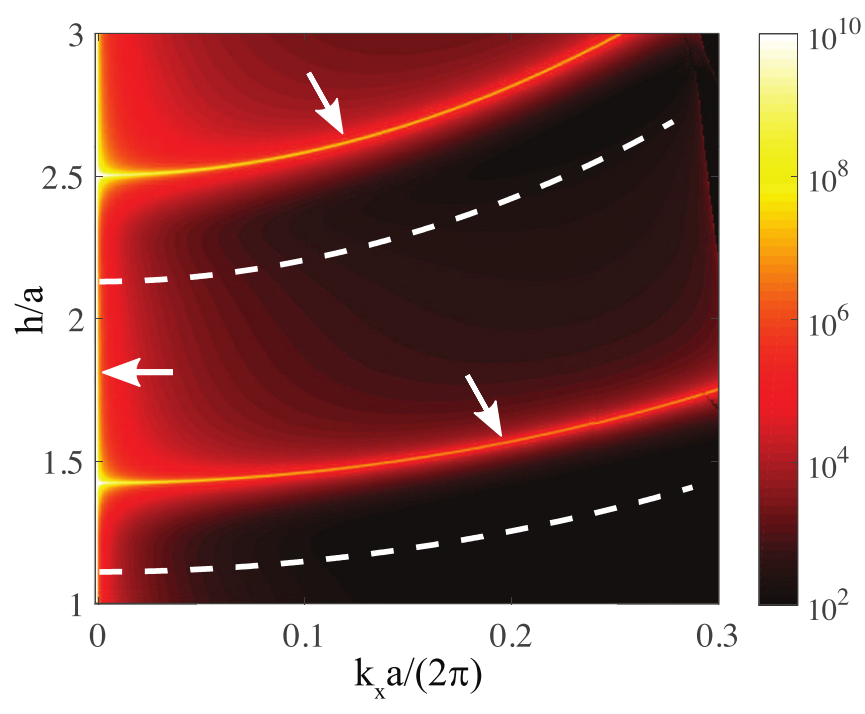

FIG. 6. Quality factor (logarithmic scale) of the green leaky mode in Fig. 2(f) as a function of $h / a$ and $k_{x} a /(2 \pi)$. The wavelength range specified during the calculations is such that only the first two BWs are propagating. The integer $p$ from Eq. (6) is set to 1, which corresponds to the green line in Figs. 2(f) and 2(g). Three branches (yellow-white color) showing an infinite $Q$ factor, and thus a BIC, are visible and shown by white arrows. Dashed white lines indicate the positions of infinite- $Q$ branches for $p=0$, which correspond to a different leaky mode also formed by the first and second BWs.

$5 / 6 \mathrm{Fa}$ and $1 / 6 \mathrm{Fa}$, respectively. Note that the total filling factor in dielectric material is preserved, $F=0.6$. Recently, a study of a PhC slab with a slot in different positions, with a fixed thickness and normal incidence angle, has been published [32]. We evidence that the symmetry-protected BICs that exist in symmetric structures at the $\Gamma$ point of the dispersion diagram becomes a resonance-trapped BIC when the horizontal mirror symmetry is broken, but only for specific values of the slab thickness.

Figure 7 shows the variation of the $Q$ factor of a leaky mode in different situations. In Fig. 7(a) we plot $Q$ as a function of the slab thickness $h$ and the asymmetry parameter $s$ for a fixed $k_{x}=0$ in a spectral range where only two BWs are propagative. We readily observe two branches that correspond to a diverging $Q$, and thus a BIC. The vertical branch for $s=0$ corresponds to the symmetry-protected BIC. As the horizontal symmetry is broken $(s \neq 0)$, the symmetry-protected BIC cannot exist anymore. Nevertheless, for specific values of the pair $(h, s)$, it becomes a resonance-trapped BIC, as evidenced by the second white branch in Fig. 7. Under the broken symmetry, $r_{12}$ no longer vanishes for $k_{x}=0$, which allows us to observe this trajectory of a resonance-trapped BIC.

Figure 7(b) displays the $Q$ factor as a function of $h$ and $s$ for $k_{x} \neq 0$. In that case, a BIC can only exist in symmetric structures for $s=0$; it is the resonance-trapped BIC labeled (2) in Fig. 2(f). This BIC disappears $\left(Q<10^{9}\right)$ when the symmetry is broken. Lastly, Fig. 7(c) displays the $Q$ factor of the same leaky mode as a function of $h$ and $k_{x}$ with a fixed asymmetry parameter $s=0.05 a$. A resonance-trapped BIC exists for $k_{x}=0$ [the one shown in Fig. 7(a)] and disappears for $k_{x} \neq 0$. These leaky modes with a finite but 

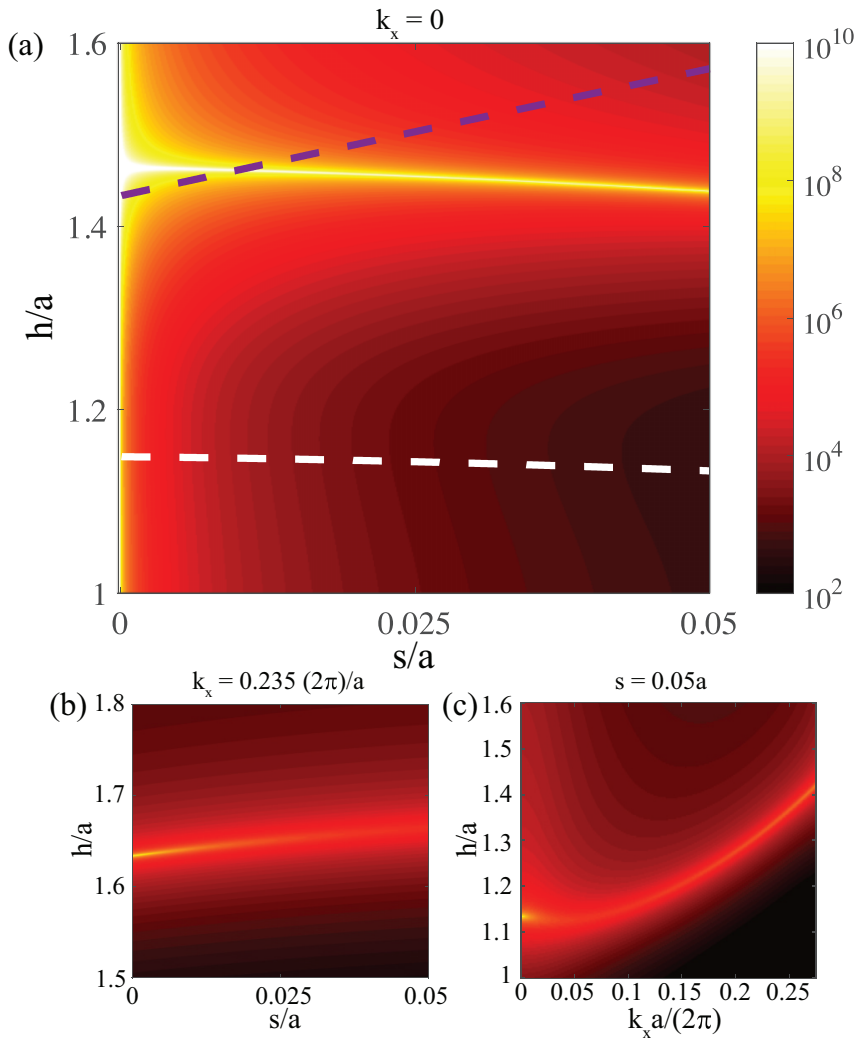

FIG. 7. Quality factor (logarithmic scale) for an asymmetric PhC slabs with two propagative BWs. (a) $Q$ factor as a function of the slab thickness $h / a$ and the asymmetry parameter $s / a$ for $k_{x}=0$. The leaky mode is calculated with $p=1$ in Eq. (6). A BIC (diverging quality factor) is clearly visible whatever the value of the asymmetry parameter. It is a symmetry-protected BIC for $s=0\left(r_{12}=0\right.$ and $\left.\left|r_{22}\right|=1\right)$ but a resonance-trapped BIC for $s \neq 0\left(r_{12} \neq 0,\left|r_{22}\right|<1\right.$, and $\left.\left|r_{\text {eff }}^{(12)}\right|=1\right)$. The dashed white line indicates the position of a similar BIC for $p=0$ and the dashed purple line indicates the position of a BIC formed by three BWs with $p=0$. (b) Same as (a), but for $k_{x} \approx 0.235(2 \pi / a)$. (c) $Q$ factor as a function of $h / a$ and $k_{x} a /(2 \pi)$ for $s=0.05 a$. Color scale and other optogeometric parameters are the same for all three subfigures.

very high- $Q$ factor are sometimes referred to as quasi-BICs in the literature [50,51].

In summary, resonance-trapped BICs can exist in asymmetric 1D PhC slabs with a broken horizontal symmetry, but only for $k_{x}=0$.

\section{CONCLUSION}

We have used a multimode Fabry-Perot model to calculate the dispersion curves and the quality factors of leaky modes supported by $1 \mathrm{D}$ symmetric and asymmetric PhC slabs. Leaky modes are transverse Fabry-Perot resonances composed of a few propagative Bloch waves bouncing back and forth vertically inside the slab. This multimode Fabry-Perot model, which does not rely on a perturbative approach, accurately predicts the existence of BICs and their positions in the parameter space regardless of the refractive index contrast. The model equally applies to symmetry-protected BICs (absence of leakage is due to symmetry incompatibility between a sin- gle BW composing the mode and the radiative plane waves) and resonance-trapped BICs (radiative leakage accidentally disappears because the contributions of several BWs interfere destructively).

The multimode Fabry-Perot model allows us to show that, regardless of the slab thickness, BICs cannot exist below a cutoff frequency, which is related to the existence of the second-order Bloch wave in the photonic crystal. In other words, BICs cannot exist in the homogenization regime. Thanks to the semianalyticity of the model, we investigate the dynamics of BICs with the slab thickness in symmetric and asymmetric photonic crystal slabs. Our calculations show that resonance-trapped BICs can exist in asymmetric 1D PhC slabs with a broken horizontal symmetry, but only for $k_{x}=0$. We evidence that the symmetry-protected BICs that exist in symmetric structures at the $\Gamma$ point of the dispersion diagram can still exist when the horizontal mirror symmetry is broken, but only for specific values of the slab thickness.

We have considered throughout the paper a filling factor $F=0.6$. The predictions of the multimode Fabry-Perot model remain as accurate for different values of $F$. The model can also be applied to 1D PhC slabs in TM polarization and to 2D PhC slabs without additional derivations. All equations presented in the paper are independent of the geometry, provided that the refractive index modulation presents vertical side walls. The only step that depends on the geometry is the numerical calculation of the propagation constants $\beta_{i}$ and the reflection coefficients $r_{i j}$ [see Fig. 3(a)]. This calculation can be done with a RCWA code [46]. Since the multimode Fabry-Perot model yields fast yet accurate predictions of the BIC location in the parameter space and provides a better understanding of the physical mechanisms that lead to the BIC formation, we think that it can become an important tool for designing $\mathrm{PhC}$ devices relying on the existence of a BIC.

\section{APPENDIX A: EXPRESSION OF $r_{\text {eff }}^{(123)}$}

The effective reflection coefficient for $N=3$ propagative Bloch waves used in Eq. (13) has the following expression:

$$
r_{\mathrm{eff}}^{(123)} \exp \left(i \beta_{3} h\right)=\frac{r_{\mathrm{eff}}^{(13)} \exp \left(i \beta_{3} h\right)+R^{(23)} \Delta+\delta_{13} \gamma}{1-R^{(23)} \gamma-\delta_{13} \Delta},
$$

with

$$
\begin{gathered}
\Delta=\alpha_{\mathrm{eff}}^{(12)} R^{(32)} r_{\mathrm{eff}}^{(12)} \exp \left(i \beta_{2} h\right)+\alpha_{\mathrm{eff}}^{(12)} \delta_{12}, \\
\gamma=\alpha_{\mathrm{eff}}^{(12)} \delta_{12} r_{\mathrm{eff}}^{(12)} \exp \left(i \beta_{2} h\right)+\alpha_{\mathrm{eff}}^{(12)} R^{(32)}, \\
R^{(23)}=\frac{r_{23}+\alpha r_{11} r_{13} r_{21} \exp \left(2 i \beta_{1} h\right)}{1-\alpha r_{13} r_{31} \exp \left[i\left(\beta_{1}+\beta_{3}\right) h\right]} \exp \left(i \beta_{2} h\right), \\
\delta_{12}=\frac{\alpha r_{12} r_{31} \exp \left[i\left(\beta_{1}+\beta_{3}\right) h\right]}{1-\alpha r_{12} r_{21} \exp \left[i\left(\beta_{1}+\beta_{2}\right) h\right]} \\
\alpha_{\mathrm{eff}}^{(12)}=\left[1-\left(r_{\mathrm{eff}}^{(12)}\right)^{2} \exp \left(2 i \beta_{2} h\right)\right]^{-1}, \\
\alpha=\left[1-r_{11}^{2} \exp \left(2 i \beta_{1} h\right)\right]^{-1} .
\end{gathered}
$$


In these expressions, the coefficient $R^{(32)}$ has the same expression as $R^{(23)}$ but with indices 2 and 3 swapped. The coefficient $\delta_{13}$ can be deduced from $\delta_{12}$ by replacing the index 2 by 3 . The effective reflection coefficients $r_{\text {eff }}^{(1 j)}$ have the same expression as in Eq. (10) with $j=2,3$ :

$$
r_{\mathrm{eff}}^{(1 j)}=\frac{r_{j j}+\alpha r_{11} r_{j 1} r_{1 j} \exp \left(2 i \beta_{1} h\right)}{1-\alpha r_{j 1} r_{1 j} \exp \left[i\left(\beta_{1}+\beta_{j}\right) h\right]} .
$$

More details on the derivation of Eq. (A1) can be found in the Supplemental Material [56].

[1] J. D. Joannopoulos, S. G. Johnson, J. N. Winn, and R. D. Meade, Photonic Crystals: Molding the Flow of light, 2nd ed. (Princeton University Press, Princeton, NJ, 2008).

[2] K. Sakoda, Optical Properties of Photonic Crystals, 2nd ed. (Springer, Berlin, 2005).

[3] M. Notomi, Rep. Prog. Phys. 73, 96501 (2010).

[4] E. Kuramochi, J. Mater. Chem. C 4, 11032 (2016).

[5] R. Baets, B. Demeulenaere, B. Dhoedt, and S. Goeman, Optical system with a dielectric subwavelength structure having high reflectivity and polarization selectivity, U. S. Patent 6191890 , 2001.

[6] C. F. R. Mateus, M. C. Y. Huang, L. Chen, C. J. ChangHasnain, and Y. Suzuki, IEEE Photonics Technol. Lett. 16, 1676 (2004).

[7] Y. Ding and R. Magnusson, Opt. Express 12, 5661 (2004).

[8] S. Hernandez, O. Gauthier-Lafaye, A. L. Fehrembach, S. Bonnefont, P. Arguel, F. Lozes-Dupuy, and A. Sentenac, Appl. Phys. Lett. 92, 131112 (2008).

[9] M. C. Y. Huang, Y. Zhou, and C. J. Chang-Hasnain, Nat. Photonics 1, 119 (2007).

[10] Y. Laaroussi, C. Chevallier, F. Genty, N. Fressengeas, L. Cerutti, T. Talercio, O. Gauthier-Lafaye, P. F. Calmon, B. Reig, J. Jacquet, and G. Almuneau, Opt. Mater. Express 3, 1576 (2013).

[11] C. Sciancalepore, B. B. Bakir, S. Menezo, X. Letartre, D. Bordel, and P. Viktorovitch, IEEE Photonics Technol. Lett. 25, 1111 (2013).

[12] Y. Park, E. Drouard, O. El Daif, X. Letartre, P. Viktorovitch, A. Fave, A. Kaminski, M. Lemiti, and C. Seassal, Opt. Express 17, 14312 (2009).

[13] M. L. Brongersma, Y. Cui, and S. Fan, Nat. Mater. 13, 451 (2014).

[14] T. Inoue, M. D. Zoysa, T. Asano, and S. Noda, Nat. Mater. 13, 928 (2014).

[15] Y. Shen, V. Rinnerbauer, I. Wang, V. Stelmakh, J. D. Joannopoulos, and M. Soljačić, ACS Photonics 2, 27 (2015).

[16] C. W. Hsu, B. Zhen, J. Lee, S.-L. Chua, S. G. Johnson, J. D. Joannopoulos, and M. Soljacic, Nature (London) 499, 188 (2013).

[17] C. Blanchard, P. Viktorovitch, and X. Letartre, Phys. Rev. A 90, 033824 (2014).

[18] C. W. Hsu, B. Zhen, A. D. Stone, J. D. Joannopoulos, and M. Soljačić, Nat. Rev. Mater. 1, 16048 (2016).

[19] F. Monticone and A. Alù, Phys. Rev. Lett. 112, 213903 (2014).

[20] P. Facchi, D. Lonigro, S. Pascazio, F. V. Pepe, and D. Pomarico, Phys. Rev. A 100, 023834 (2019).

[21] J. von Neumann and E. P. Wigner, Phys. Z. 30, 465 (1929).

[22] H. Friedrich and D. Wintgen, Phys. Rev. A 32, 3231 (1985).
[23] J. W. Yoon, S. H. Song, and R. Magnusson, Sci. Rep. 5, 18301 (2015).

[24] C. Blanchard, J.-P. Hugonin, and C. Sauvan, Phys. Rev. B 94, 155303 (2016).

[25] Z. F. Sadrieva, I. S. Sinev, K. L. Koshelev, A. Samusev, I. V. Iorsh, O. Takayama, R. Malureanu, A. A. Bogdanov, and A. V. Lavrinenko, ACS Photonics 4, 723 (2017).

[26] A. Kodigala, T. Lepetit, Q. Gu, B. Bahari, Y. Fainman, and B. Kanté, Nature (London) 541, 196 (2017).

[27] S. T. Ha, Y. H. Fu, N. K. Emani, Z. Pan, R. M. Bakker, R. Paniagua-Domínguez, and A. I. Kuznetsov, Nat. Nanotechnol. 13, 1042 (2018).

[28] Y. Liu, W. Zhou, and Y. Sun, Sensors 17, 1861 (2017).

[29] S. Romano, G. Zito, S. Torino, G. Calafiore, E. Penzo, G. Coppola, S. Cabrini, I. Rendina, and V. Mocella, Photon. Res. 6, 726 (2018).

[30] F. Yesilkoy, E. R. Arvelo, Y. Jahani, M. Liu, A. Tittl, V. Cevher, Y. Kivshar, and H. Altug, Nat. Photonics 13, 390 (2019).

[31] L. Li and H. Yin, Sci. Rep. 6, 26988 (2016).

[32] Y. Wang, J. Song, L. Dong, and M. Lu, J. Opt. Soc. Am. B 33, 2472 (2016).

[33] X. Gao, C. W. Hsu, B. Zhen, X. Lin, J. D. Joannopoulos, M. Soljačić, and H. Chen, Sci. Rep. 6, 31908 (2016).

[34] S. I. Azzam, V. M. Shalaev, A. Boltasseva, and A. V. Kildishev, Phys. Rev. Lett. 121, 253901 (2018).

[35] E. N. Bulgakov, D. N. Maksimov, P. N. Semina, and S. A. Skorobogatov, J. Opt. Soc. Am. B 35, 1218 (2018).

[36] Y. Yang, C. Peng, Y. Liang, Z. Li, and S. Noda, Phys. Rev. Lett. 113, 037401 (2014).

[37] Z. Wang, H. Zhang, L. Ni, W. Hu, and C. Peng, IEEE J. Quantum Electron. 52, 1 (2016).

[38] L. Ni, Z. Wang, C. Peng, and Z. Li, Phys. Rev. B 94, 245148 (2016).

[39] E. N. Bulgakov and D. N. Maksimov, Phys. Rev. A 98, 053840 (2018).

[40] S. Fan, W. Suh, and J. D. Joannopoulos, J. Opt. Soc. Am. A 20 , 569 (2003).

[41] A.-L. Fehrembach and A. Sentenac, J. Opt. Soc. Am. A 20, 481 (2003).

[42] E. Popov, L. Mashev, and D. Maystre, Opt. Acta 33, 607 (1986).

[43] A.-L. Fehrembach, D. Maystre, and A. Sentenac, J. Opt. Soc. Am. A 19, 1136 (2002).

[44] J. M. Pottage, E. Silvestre, and P. S. J. Russell, J. Opt. Soc. Am. A 18, 442 (2001).

[45] T. Clausnitzer, T. Kämpfe, E. B. Kley, A. Tünnermann, U. Peschel, A. V. Tishchenko, and O. Parriaux, Opt. Express 13, 10448 (2005).

[46] P. Lalanne, J. P. Hugonin, and P. Chavel, J. Lightwave Technol. 24, 2442 (2006). 
[47] V. Karagodsky, F. G. Sedgwick, and C. J. Chang-Hasnain, Opt. Express 18, 16973 (2010).

[48] V. Karagodsky, C. Chase, and C. J. Chang-Hasnain, Opt. Lett. 36, 1704 (2011).

[49] V. Karagodsky and C. J. Chang-Hasnain, Opt. Express 20, 10888 (2012).

[50] D. A. Bykov, E. A. Bezus, and L. L. Doskolovich, Phys. Rev. A 99, 063805 (2019).

[51] K. Koshelev, S. Lepeshov, M. Liu, A. Bogdanov, and Y. Kivshar, Phys. Rev. Lett. 121, 193903 (2018).

[52] M. G. Moharam, E. B. Grann, D. A. Pommet, and T. K. Gaylord, J. Opt. Soc. Am. A 12, 1068 (1995).

[53] Q. Bai, M. Perrin, C. Sauvan, J.-P. Hugonin, and P. Lalanne, Opt. Express 21, 27371 (2013).

[54] P. Lalanne, W. Yan, A. Gras, C. Sauvan, J.-P. Hugonin, M. Besbes, G. Demésy, M. D. Truong, B. Gralak, F. Zolla, A. Nicolet, F. Binkowski, L. Zschiedrich, S. Burger, J.
Zimmerling, R. Remis, P. Urbach, H. T. Liu, and T. Weiss, J. Opt. Soc. Am. A 36, 686 (2019).

[55] P. Lalanne, S. Astilean, P. Chavel, E. Cambril, and H. Launois, J. Opt. Soc. Am. A 16, 1143 (1999).

[56] See Supplemental Material at http://link.aps.org/supplemental/ 10.1103/PhysRevB.101.155303 for more details on the derivation of the effective reflection for $N=2$ and 3 waves and for additional calculations.

[57] G. Lecamp, P. Lalanne, J. P. Hugonin, and J. M. Gérard, IEEE J. Quantum Electron. 41, 1323 (2005).

[58] H. A. Haus, Waves and Fields in Optoelectronics (Prentice-Hall, London, 1984).

[59] P. Lalanne, C. Sauvan, and J. P. Hugonin, Laser Photon. Rev. 2, 514 (2008).

[60] C. Sauvan, P. Lalanne, and J. P. Hugonin, Phys. Rev. B 71, 165118 (2005). 